\title{
Nicht ossifizierendes Knochenfibrom und benigne fibröse Histiozytome: ein Update
}

Nicht ossifizierende Knochenfibrome (NOF) sind gutartige und selbstlimitierende Läsionen, die gemäß aktueller WHO-Klassifikation in $30-40 \%$ der Bevölkerung während der Skelettreifung entstehen und wieder verschwinden. Sie kommen typischerweise an den Metaphysen der langen Röhrenknochen vor und können gelegentlich auch multifokal ausgebildet sein ( $\triangleright$ Abb. 1a und b).

Im Regelfall ist die Bildgebung dieser asymptomatischen und gewöhnlich als ZufalIsbefund auffallenden Läsionen so charakteristisch, dass eine Diagnose auch ohne histologische Absicherung zuverlässig möglich ist. NOF zählen daher zu den sog. „Don't-touch“-Befunden, bei denen eine Biopsie nicht angezeigt ist. Frühstadien kennzeichnen sich in der Bildgebung durch eine Kortikalis-zentrierte Aufhellung, deren Längsachse parallel zur Achse des befallenen Röhrenknochens ausgerichtet ist. Man spricht in diesen frühen Stadien auch vom fibrösen Kortikalisdefekt. Wenn sich der Befund vergrößert und in den Markraum erstreckt, erkennt man im konventionellen Röntgenbild traubenförmig konfigurierte Osteolysen, die von einem sklerotischen Randsaum umgeben werden. In der Regel handelt es sich um Lodwick Grad IA Befunde.

In seltenen Fällen können NOF so groß werden, dass sie die biomechanische Stabilität des Knochens gefährden und zu einer pathologischen Fraktur führen. In dieser Konstellation wird die Läsion in der Regel kürettiert und vom Pathologen histologisch untersucht. Hierbei kommen storiforme Spindelzellproliferationen in Kombination mit Siderophagen, osteoklastären Riesenzellen sowie Schaumzellakkumulationen zur Darstellung. Es besteht eine auffallende morphologische Ähnlichkeit mit zentralen Riesenzellgranulomen des Kiefers.
NOF wurden lange Zeit als reaktive Läsionen und nicht als echte Neoplasien angesehen, v. a. bedingt durch ihren selbstlimitierenden Verlauf. Eine Panel-Sequenzierung eines Konsilfalls zeigt überraschenderweise eine KRAS-Mutation eines radiologisch und histologisch typischen Falls, weshalb ein Kollektiv von 19 NOF mit Frischgewebsproben Exomsequenziert und weitere 40 NOF mit Paraffingewebe Panel-sequenziert wurde. Tatsächlich zeigte sich in 38 von 59 (64,4\%) Fällen eine klar pathogene Hotspot-Mutation im KRAS-Gen und in acht weiteren Fällen (13,6\%) eine FGFR1-Mutation ( $\triangleright$ Abb. 2). Zusätzlich wurden in zwei Fällen NF1-Mutationen detektiert, bei denen aber klinisch bereits eine Neurofibromatose Typ 1 bekannt war [1].

Tatsächlich wurden pathogene KRAS-Mutationen zuvor auch in anderen benignen Erkrankungen beschrieben, z. B. in arterio- 

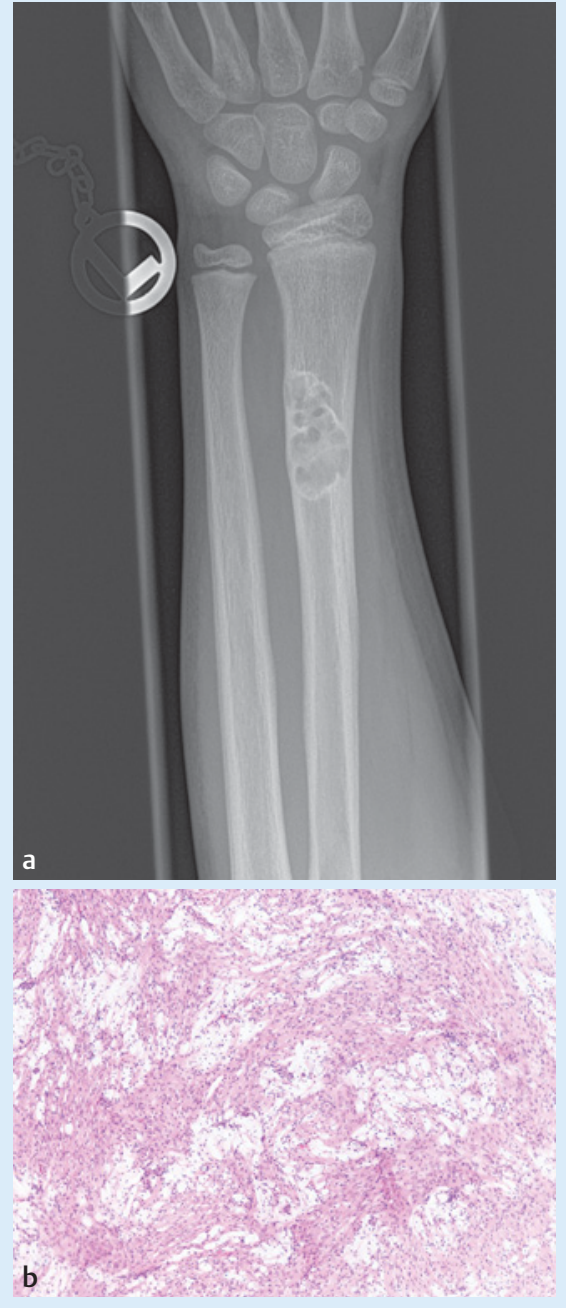

Abb. 1 Nicht ossifizierendes Knochenfibrom des distalen Radius mit traubenförmiger und randsklerosierter Osteolyse (a). Die Histologie zeigt storiforme Spindelzellproliferate ohne zelluläre Atypien und ausgedehnte Schaumzellakkumulationen (b).

venösen Malformationen des Gehirns und in benignen Endometrioseherden [2, 3]. Der Nachweis entsprechender Mutationen in NOF (gesamthaft in 48 von 59 untersuchten NOF, 81,4\%) kann als Argument dafür gewertet werden, dass es sich doch um echte Neoplasien handelt. Außerdem passt das gehäufte Vorkommen im Rahmen der Neurofibromatose Typ 1 dazu, dass NOF zum Spektrum von Erkrankungen zählen, die mit Mutationen im MAP-Kinase Signalweg einhergehen (sog. RASopathien). Der selbstlimitierende Verlauf der Läsionen bleibt pathogenetisch ungeklärt. Möglicherweise kommt es im Verlauf zu einem Ausdünnen der mutationstragenden Zellen durch Apoptose, wie es z.B. auch bei der fibrösen
Dysplasie aufgezeigt werden konnte, sodass der proliferative Stimulus ausbrennt [4]. Interessanterweise konnten vor kurzem auch in zentralen Riesenzellgranulomen in einem hohen Prozentsatz KRAS- und FGFR1-Mutationen nachgewiesen werden, die damit nicht nur morphologisch, sondern auch von ihrem genetischen Hintergrund Gemeinsamkeiten mit NOF aufweisen [5].

Benigne fibröse Histiozytome (BFH) werden in der aktuellen WHO-Klassifikation noch im gleichen Kapitel wie NOF abgehandelt und sind definiert als Läsionen mit identischer Morphologie, aber atypischer Lokalisation, wobei gehäuft eine epiphysäre Lage beschrieben wurde [6]. Neuere Untersuchungen konnten allerdings zeigen, dass in einem Großteil der als BFH bezeichneten Läsionen H3F3A-Mutationen (p.G34W) nachweisbar sind und es sich daher um regressiv veränderte Riesenzelltumoren des Knochens handelt. In der voraussichtlich Ende dieses Jahres oder Anfang des nächsten Jahres erscheinenden 5. Auflage der WHO-Klassifikation für Knochenund Weichteiltumoren werden die BFH daher nicht mehr als eigenständige Entität aufgeführt, sondern im Rahmen der Riesenzelltumoren beschrieben.

\section{Autorinnen / Autoren}

\section{Prof. Dr. med. Daniel Baumhoer}

Knochentumor-Referenzzentrum am Institut für Pathologie, Universitätsspital Basel Schweiz

Korrespondenzadresse

Prof. Dr. med. Daniel Baumhoer

Knochentumor- und DÖSAK-ReferenzzentrumInstitut für Medizinische Genetik und Pathologie

Universitätsspital Basel

Schönbeinstrasse 40

$\mathrm{CH}-4031$ Basel

Switzerland

E-Mail: daniel.baumhoer@usb.ch

\section{Literatur}

[1] Baumhoer D, Kovac M, Sperveslage J et al. Activating mutations in the MAP-kinase pathway define non-ossifying fibroma of bone. J Pathol 2019; 248: 116-122

[2] Anglesio MS, Papadopoulos N, Ayhan A et al. Cancer-Associated Mutations in Endometriosis without Cancer. N Engl J Med 2017; 376: 1835 1848

[3] Nikolaev SI, Vetiska S, Bonilla X et al. Somatic Activating KRAS Mutations in Arteriovenous Malformations of the Brain. N Engl J Med 2018; 378: 250-261

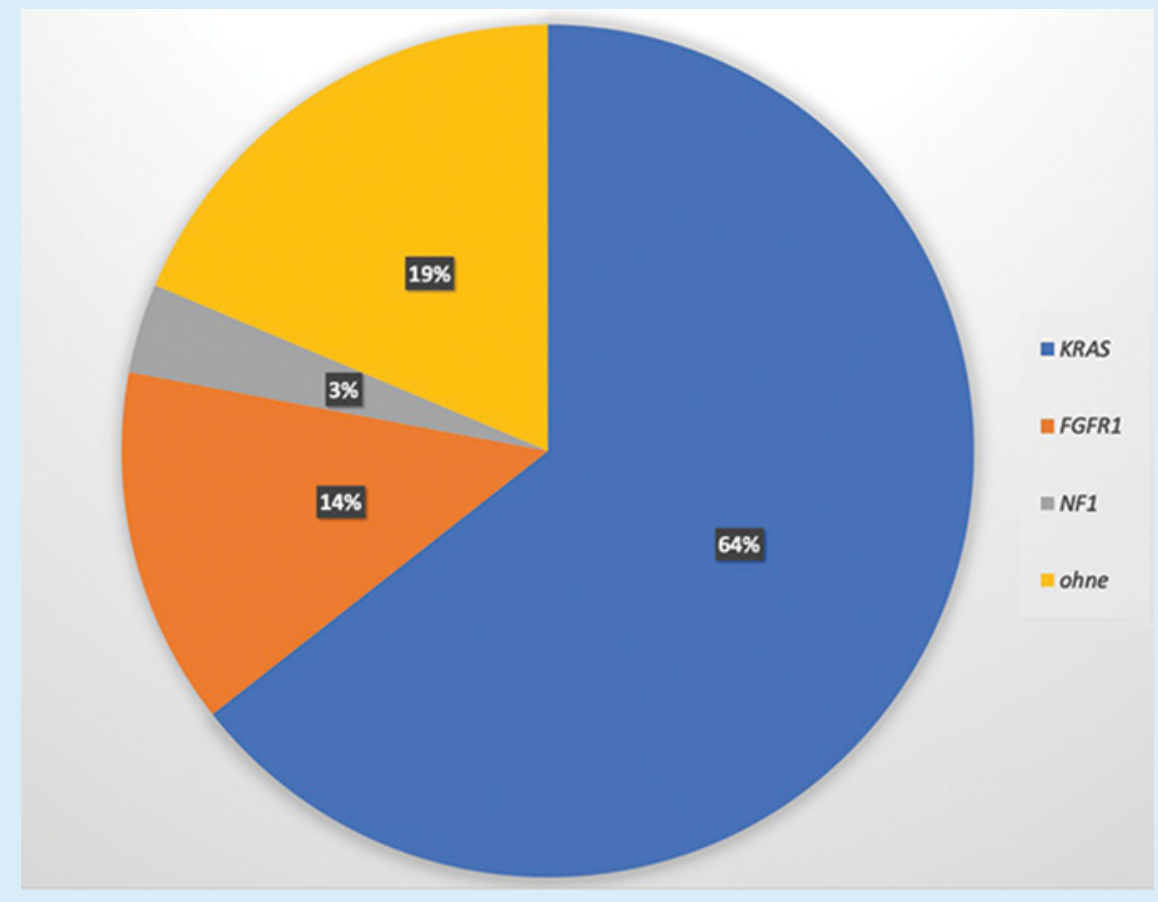

Abb. 2 Mutationen im MAP-Kinase Pathway bei nicht ossifizierenden Knochenfibromen ( $n=59)$. 
[4] Kuznetsov SA, Cherman N, Riminucci M et al. Age-dependent demise of GNAS-mutated skeletal stem cells and "normalization" of fibrous dysplasia of bone. J Bone Miner Res 2008; 23: 1731-1740
[5] Gomes CC, Gayden T, Bajic A et al. TRPV4 and KRAS and FGFR1 gain-of-function mutations drive giant cell lesions of the jaw. Nat Commun 2018; 9: 45-72
[6] Matsuno T. Benign fibrous histiocytoma involving the ends of long bone. Skeletal Radiol 1990; 19: 561-566 